\title{
Determination of pressure data from velocity data with a view towards its application in cardiovascular mechanics, Part 1: Theoretical considerations
}

\begin{abstract}
The non-invasive determination of the pressure (normal stress) in a flowing fluid has ramifications in a variety of important problems: the flow of blood in blood vessels, and flows taking place in inaccessible locations in complex internal geometries that occur in mechanical systems. In this paper we discuss a rigorous new mathematical procedure for the determination of the pressure (normal stress) field, from data for the velocity field that can be obtained through imaging procedures such as 4D Magnetic Resonance Imaging or Echocardiography. We then use the procedure to demonstrate its efficacy by considering flows in an idealized geometry with a symmetric and asymmetric obstruction. We delineate the superiority of the method with regard to the methods that are currently in place. In Part II of this two part paper, we study the loss of pressure and the dissipation that occurs due to the flow of blood across a diseased valve (the pressure loss being an important indicator of the extent of the valvular disease) as well as the flow taking place in a realistic cerebral aneurysm.
\end{abstract}

Keywords: Non-invasive pressure determination, Pressure-Poisson Equation, Stokes Equation, Heart valve, blood vessel.

\section{Introduction}

The ability to non-invasively determine the pressure in a flowing fluid has wide ranging technological relevance and import, an example being problems in medicine concerning the flow of biological fluids. The cardio-vascular system presents several situations wherein the non-invasive determination of pressure would significantly reduce serious risks, including fatality, associated with invasive procedures. This two part study is concerned with the determination of the pressure field from non-invasive velocity data with a view towards determining the pressure drop across a diseased valve as a consequence of the dissipation in the fluid as it flows through the valve, the loss of pressure bearing a direct relation to the extent of the disease. While there have been some careful mathematical attempts at determining the pressure field from information for the velocity field, with regard to the Navier-Stokes fluid (see the references below), most of the studies concerning the determination of pressure from the velocity data with regard to flow across diseased valves and other related cardiovascular flow problems are based on an appeal to inappropriate governing equations, namely the Bernoulli equation, which are grossly inadequate to describe the dissipation that takes place in a flowing fluid. This is usually addressed by an ad hoc modification to the Bernoulli 
equation by adding a dissipation term; see Akins et al. (2008). What is however required is a much more careful consideration of the viscous dissipation that takes place during the fluid flow and the resulting pressure drop. We discuss in detail the inverse problem of determining the pressure field from data for the velocity field which might or might not be known precisely. The study does not appeal to the Bernoulli equation but considers in full the Navier-Stokes equations for an incompressible linearly viscous fluid. In Part 1 of the study, in order to clarify and simplify the procedure, we consider the flow in an idealized geometry of the stenotic aortic valve. In Part 2 we will present broader study with determining the dissipation directly from the model. Part 2 will conclude the results, amount of dissipation and corresponding difference of the pressure drop, in geometries with different levels of the severity up to $85 \%$ in symmetric stenotic valves and we also present some results concerning the determination of pressure in flows occurring in cerebral aneurysms in realistic geometries. In Part 1, we provide the details of the mathematical issues and the numerical procedures that are adopted to obtain the pressure field from the velocity field, while the motivation for the consideration of the problem of Valvular stenosis and Valvular disease is provided in Part 2.

While blood in small vessels exhibits shear-thinning characteristics, in a vessel of the size under consideration it can be modeled as a Navier-Stokes fluid. The resulting equations of the pressure determination can be expressed in two different ways, one as a Poisson equation for the pressure field and the other based on the Stokes equation with additional stabilization/correction term. The former method even in the weak form requires higher derivatives than the latter method. In order to ascertain the efficiency of the two methods we compute the pressure field corresponding to three types of velocity data, the first which we refer to as "fine data" wherein we have full information for the velocity field on a very fine grid, the second type of data which we refer to as "coarse data" wherein the information is available only on a rough grids with different level of the coarsness, and finally the third type of data, "data with a noise", wherein the first two types are combined with an uncertainty in its values. We compare the results for the pressure field obtained from the three types of data for the velocity field using the two different methods, the pressure Poisson equation (PPE) method and the Stokes equation (STE) method. To our knowledge such a comparative study of the numerical schemes that delineates the differences in the results from the two schemes and which clearly indicates the STE method to be superior to the PPE method, for the problem on hand have not been carried out.

We idealize the geometry of the flow to a flow in a rigid pipe with an obstruction knowing full well that the real flow domain is far more complicated and the walls being highly deformable. Our aim is to solve eventually such a complex problem for the determination of the pressure field from the data for the velocity field. While we have some experience in the numerical resolution of problems concerning fluid-solid interactions with regard to a direct determination of the velocity and pressure fields, see Hron and Madlik (2007), Razzaq et al. (2012), we have to develop the codes in the case of the problem of determining the pressure field from a knowledge of the velocity field. In Part 2 of the paper we consider the flow in the actual geometry that matches anatomical conditions. In this Part 1, our aim is to establish the proof of concept with regard to the mathematical formulation and the numerical procedure. We 
do use physiologic data for the inlet and outlet condition upstream and downstream of the obstruction (stenosis) but the flow geometry is idealized.

\subsection{Determining/Ascertaining the pressure for the flow of the Navier-Stokes fluid}

The pressure loss across a diseased valve or stenotic artery is thought to be due to the dissipation that takes place during the flow across the valve. In view of this, there has been a great deal of effort expended in determining the dissipation due to the flow across a diseased valve; see Akins et al. (2008), see Dasi et al. (2009). However, all these efforts are totally ad hoc and appeal to procedures that are clearly not suited for evaluating the dissipation. The attempts at describing the energy losses across a diseased valve in studies like those carried out by Akins et al. (2008) and Dasi et al. (2009), ignore the fact that the blood vessel is a deformable medium that is in fact undergoing large deformations which would lead to a flow that is very different from that which takes place in a rigid pipe. (Our study also suffers from the same drawback.) The walls of the blood vessel are in fact inhomogeneous and anisotropic, and more importantly viscoelastic. Thus, in order to determine the flow characteristic of blood across a diseased valve, it is necessary to solve the equations that couple the motion of the wall boundary as well as the equations governing the flowing blood. The problem is exceedingly difficult in view of the fact that the flow domain is changing with time and the domain itself can only be determined by solving the coupled system of equations. It is our aim to eventually study such a problem.

In very small blood vessels such as capillaries as the diameter of the red blood cells are of comparable or even larger diameter than that of the blood vessel, even using a continuum fluid model for blood is totally inappropriate. However, in the problem of flow across a diseased valve or arterial stenosis the flow of blood can be well described by the classical incompressible Navier-Stokes fluid. Even if we consider the fluid flowing in a vessel that has a rigid boundary, the problem is quite challenging as we have to solve the problem wherein locally the Reynolds number is very large, the geometry of the flow domain complex, and the flow three dimensional and unsteady.

Let us suppose that blood can be described by the Navier-Stokes constitutive relation, that is

$$
\mathbf{T}=-p \mathbf{I}+2 \mu \mathbf{D},
$$

where $\mathbf{T}$ is the Cauchy stress, $p=-\frac{1}{3}(\operatorname{tr} \mathbf{T})$ is the mean normal stress that is usually referred to as the mechanical pressure (see Rajagopal (2015) for a detailed discussion of the notion of "pressure"), $\mu$ is the dynamic viscosity and $\left.\mathbf{D}=\frac{1}{2}\left(\nabla \mathbf{v}+(\nabla \mathbf{v})^{T}\right)\right)$. Since the fluid is incompressible, it can only undergo isochoric motions so that the constraint

$$
\operatorname{tr} \mathbf{D}=\operatorname{div} \mathbf{v}=0
$$

is satisfied. We shall assume that the fluid is homogeneous and hence the balance of mass reduces to the density $\varrho$ being a constant everywhere. 
On neglecting body forces and substituting the constitutive relation (1) into the balance of linear momentum

$$
\varrho \frac{\mathrm{d} \mathbf{v}}{\mathrm{d} t}=\operatorname{div} \mathbf{T},
$$

where $\varrho$ is the density, and $\frac{\mathrm{d}}{\mathrm{d} t}$ is the material time derivative given by

$$
\frac{\mathrm{d} \mathbf{v}}{\mathrm{d} t}=\frac{\partial \mathbf{v}}{\partial t}+(\nabla \mathbf{v}) \mathbf{v}
$$

and on dividing by the constant $\varrho$ we obtain the Navier-Stokes equation for an incompressible fluid, namely

$$
\frac{\partial \mathbf{v}}{\partial t}+(\nabla \mathbf{v}) \mathbf{v}+\nabla \frac{p}{\varrho}-\operatorname{div}(2 v \mathbf{D}(\mathbf{v}))=0 \quad \forall(\mathbf{x}, t) \in \Omega \times \mathcal{R}
$$

with kinematic viscosity $v=\frac{\mu}{\varrho}$.

Now instead of trying to get some sort of an estimate on the loss of pressure as a consequence of the dissipation, we discuss in Sec. 1.1 how to directly obtain the pressure field once the velocity field is known. The velocity field is assumed to have been determined by either echocardiography or 4D Magnetic Resonance Imaging, and we will consider both knowledge of the velocity field at a limited amount of the points and the possibility of uncertainty of the values for the velocity field.

In general one would like to find solution to the Navier-Stokes systems (5) and (2), with velocity as close as possible to the available velocity measurement. However, let us initially assume we know the velocity field possibly satisfying (2) and we look for the pressure $p$ such that the pair $(\mathbf{v}, p)$ solves also (5). In biomedical context this has been investigated for example in Heys et al. (2010); Gurka et al. (1999); Yang et al. (1996); Krittian et al. (2012); Bolin and Raguin (2008); Charonko et al. (2010); Song et al. (1994); Dabiri et al. (2014). See also Van Oudheusden (2013) for an overview of other areas than biofluid mechanics where the problem of pressure recovery from measured velocity data is investigated.

In all of the cited references, the PPE method is used to compute the pressure. We compare the PPE method, as described in Sec.1.1.1. with a new approach, namely the STE method as presented in Sec. 1.1.2 The STE method is based on the Helmholtz decomposition theorem (see for example Maria Denaro (2003) for its application in the context of projection methods), and it allows us to compute the pressure under lower regularity requirements on the given velocity. In Cayco and Nicolaides (1986) this aproach is used to recover pressure for two-dimensional flows that allows the authors to use the stream function formulation. However we have not found any publication using this method to recover the pressure for given velocity field in general three dimensional flows. In Sec. 2 we show that the STE method provides more accurate pressure approximation than PPE method for the same velocity data. Of course, in the STE method we solve a system of four partial differential equations in three dimensions, while the PPE method requires solving just one scalar equation.

In general one can try to solve a constraint optimization problem of the type: find the couple $(\mathbf{w}, q)$ such that it satisfies the Navier-Stokes equation inside the domain $\Omega$ 
and simultaneously the velocity field $\mathbf{w}$ is close to the measured velocity $\mathbf{v}$ in some sense. this has been considered for example in Heys et al. (2010) where the Galerkin least squares method (GLS) is used to recover the pressure from PIV measurements. In this case, there is no need to compute any derivatives of the given (i.e. measured) velocity field. On the other hand one has to solve symmetric, positive definite, but much larger and nonlinear system of equations. in this study we will restrict to the linear methods.

\subsubsection{Determination of the pressure by the PPE method}

We now start with a discussion of the PPE method. We can derive from (5) for $q_{\mathrm{ppe}} \approx p$ that

$$
-\nabla q_{\mathrm{ppe}}=\mathbf{f}(\mathbf{v})
$$

where we define the quantity

$$
\mathbf{f}(\mathbf{v})=\varrho \frac{\partial \mathbf{v}}{\partial t}+\varrho(\nabla \mathbf{v}) \mathbf{v}-\operatorname{div}(2 \mu \mathbf{D}(\mathbf{v}))
$$

which depends only on the given velocity field $\mathbf{v}$.

On taking the divergence of Navier-Stokes equations (5) we obtain, in virtue of incompressibility condition (2), that

$$
-\Delta p=\varrho \nabla \mathbf{v} \cdot(\nabla \mathbf{v})^{T} .
$$

Since the real problem takes place in a complex geometry, we will have to solve the Laplace's equation (8) numerically. For a given velocity field $\mathbf{v}$, which may not be necessarily divergence free and consequently $\operatorname{div} \mathbf{f}(\mathbf{v})$ differs from the right hand side of $\left[8\right.$, we want to solve the problem for $q_{\mathrm{ppe}} \approx p$, where $q_{\mathrm{ppe}}$ meets

$$
\begin{aligned}
-\Delta q_{\mathrm{ppe}} & =\operatorname{div} \mathbf{f}(\mathbf{v}), \\
\frac{\partial q_{\mathrm{ppe}}}{\partial \mathbf{n}} & =-\mathbf{f}(\mathbf{v}) \cdot \mathbf{n}
\end{aligned}
$$

with $\mathbf{f}(\mathbf{v})$ given by (7). We approximate $\frac{\partial \mathbf{v}}{\partial t}$ in (7) by $\frac{\mathbf{v}-\mathbf{v}^{k}}{t^{k+1}-t^{k}}$ where $\mathbf{v}$ is a given velocity field at current time step $t^{k+1}$ and $\mathbf{v}^{k}$ is (also given) velocity field at previous time step $t^{k}$.

Since this problem admits unique solution up to a constant we need to supply some additional condition to have uniquely defined solution. We will provide a priori knowledge of the pressure mean value over the outlet of the domain.

We refer to the problem of finding a numerical solution of (9) and (10) with (7) and this additional condition fixing the value of pressure as the PPE method.

This method is described for example in Heys et al. (2010); Gurka et al. (1999); Yang et al. (1996); Krittian et al. (2012); Bolin and Raguin (2008); Charonko et al. (2010); Song et al. (1994); Dabiri et al. (2014); Shirokoff and Rosales (2011) and is closely connected to a projection step in the projection methods used to decouple the 
velocity-pressure computation when solving the Navier-Stokes equations, see for example Liu et al. (2010); Prohl (2008); Guermond et al.(2006). In Song et al. (1994) it is shown, that the PPE method is equivalent to finding pressure $p$ such that the functional $\|\nabla p-\mathbf{f}(\mathbf{v})\|_{\Omega}^{2}$ is minimized.

Equation (9) is a second order partial differential equation. In order to solve (9) for $p$, we need to know the appropriate boundary conditions for $p$. Boundary conditions are determined by the physics of the problem. However, when one is unable to determine the appropriate boundary conditions based on an understanding of the physics of the problem, one can take recourse to a mathematical procedure that is reasonable and expected of the solution to the problem, provided one is assured of a solution that is sufficiently smooth. One can obtain conditions by evaluation the governing partial differential equation on the boundary.

This method leads to a scalar linear problem. However even in the weak form it requires computation of first or even second derivatives of the velocity field, depending how many derivatives we can move to the test functions, which can be problematic if the velocity field is measured.

\subsubsection{Determination of the pressure using the STE method}

In this approach, based on the idea from Cayco and Nicolaides (1986), we want to directly determine a pressure as a function $q_{\text {ste }}$ such that $-\nabla q_{\text {ste }}=\mathbf{f}(\mathbf{v})$ in a weak sense, where $\mathbf{f}(\mathbf{v})$ is given by (7).

This is done by solving a Stokes problem for the unknowns $\mathbf{w}$ and $q_{\text {ste }} \approx p$ such that

$$
\begin{aligned}
-\Delta \mathbf{w}-\nabla q_{\text {ste }} & =\mathbf{f}(\mathbf{v}) & & \text { in } \Omega, \\
\operatorname{div} \mathbf{w} & =0 & & \text { in } \Omega, \\
\mathbf{w} & =\mathbf{0} & & \text { on } \Gamma .
\end{aligned}
$$

It is important to recognize that $\mathbf{w}$ is considered here as an unknown and has nothing common with the given velocity $\mathbf{v}$. In fact, $\mathbf{w}$ is expected to be almost zero. Again we need to provide a priori knowledge of the pressure at one point, or pressure mean value over some part of the domain or its boundary, to get a unique solution.

The solution of (11)-(13) with the right hand side of (7) and the prescribed mean pressure value over the outlet is referred to as the STE method.

This method leads to a larger linear saddle-point type system of equations. However it requires less regularity on the data than the PPE method introduced in the previous section since no additional derivative of the data vector $\mathbf{f}(\mathbf{v})$ is needed.

\subsubsection{Weak formulation of the problems}

The subsequent computations are run using the automated finite element solver Logg et al. (2012) which allows for an easy employment of the standard finite element method to a wide class of variational problems. First, the Navier-Stokes problem (5) \& (2) is written in variational form as: 
Find $(\mathbf{v}, p) \in V \times P$ such that for all $(\mathbf{u}, q) \in V \times P$

$$
\begin{gathered}
\int_{\Omega} \frac{\partial \mathbf{v}}{\partial t} \cdot \mathbf{u} \mathrm{d} x+\int_{\Omega}(\nabla \mathbf{v}) \mathbf{v} \cdot \mathbf{u} \mathrm{d} x=\frac{1}{\varrho} \int_{\Omega} \mathbf{T}: \nabla \mathbf{u} \mathrm{d} x, \\
\int_{\Omega} q \operatorname{div} \mathbf{v} \mathrm{d} x=0 .
\end{gathered}
$$

The discretization in time is done by an implicit one step difference method and with regard to spatial discretization, the function spaces $V, P$ are approximated by standard piecewise polynomial spaces, i.e. Taylor-Hood $P_{2} / P_{1}$ elements.

The PPE and STE problems are cast in weak forms as follows:

PPE Find $\left(q_{\mathrm{ppe}}, r\right) \in P_{P} \times \mathcal{R}$ such that for all $(\widehat{q}, \widehat{r}) \in P_{P} \times \mathcal{R}$

$$
\begin{aligned}
& \int_{\Omega} \nabla q_{\mathrm{ppe}} \cdot \nabla \widehat{q} \mathrm{~d} x=\int_{\Omega} \mathbf{f} \cdot \nabla \widehat{q}+\int_{\Gamma_{\text {out }}} \widehat{q} \widehat{\mathrm{d}} s, \\
& \int_{\Gamma_{\text {out }}}\left(q_{\mathrm{ppe}}-p\right) \widehat{r} \mathrm{~d} s=0 .
\end{aligned}
$$

STE Find $\left(\mathbf{w}, q_{\text {ste }}, r\right) \in V \times P_{S} \times \mathcal{R}$ such that for all $(\widehat{\mathbf{w}}, \widehat{q}, \widehat{r}) \in V \times P_{S} \times \mathcal{R}$

$$
\begin{aligned}
\int_{\Omega} \nabla \mathbf{w}: \nabla \widehat{\mathbf{w}} \mathrm{d} x-\int_{\Omega} q_{\mathrm{ste}} \operatorname{div} \widehat{\mathbf{w}} \mathrm{d} x & =\int_{\Omega} \mathbf{f} \cdot \widehat{\mathbf{w}} \mathrm{d} x, \\
\int_{\Gamma_{\text {out }}} \widehat{r q} \mathrm{~d} s+\int_{\Omega} \widehat{q} \operatorname{div} \mathbf{w} \mathrm{d} x & =0 \\
\int_{\Gamma_{\text {out }}}\left(q_{\mathrm{ste}}-p\right) \widehat{r} \mathrm{~d} s & =0 .
\end{aligned}
$$

Standard FEM discretization is used to find the approximate solution to these problems. The space $P_{S}$ is approximated by piecewise $P_{1}$ functions and the spaces $P_{P}$ and $V$ by piecewise $P_{2}$ functions. The space $\mathcal{R}$ is one dimensional space of constants on the computational domain and is used to enforce the additional condition on the mean value of the pressure over the $\Gamma_{\text {out }}$ part of the boundary by means of global Lagrange multiplier.

\section{Numerical results}

In Sec. 1.1.1 and 1.1.2 we introduced two approaches to ascertain the pressure directly from the velocity field. In order to compute the results achieved by these two methods we introduce the reference velocity field $\mathbf{v}_{\text {ref }}$ given as a numerical solution of the incompressible Navier-Stokes equations. This velocity field $\mathbf{v}_{\text {ref }}$ will serve as the input data for PPE and STE methods, and we refer to $\mathbf{v}_{\text {ref }}$ as a reference flow. The computed pressure $p_{\text {ref }}$ will be used as the reference pressure used to compare the accuracy of these two methods. 


\subsection{Reference flow}

In a given domain $\Omega \subset \mathbf{R}^{3}$, representing the simplified aortic valve, the velocity $\mathbf{v}_{\text {ref }}$ and pressure $p_{\text {ref }}$ satisfy

$$
\begin{array}{r}
\frac{\partial \mathbf{v}_{\text {ref }}}{\partial t}+\left(\nabla \mathbf{v}_{\text {ref }}\right) \mathbf{v}_{\text {ref }}+\nabla \frac{p_{\text {ref }}}{\varrho}-\operatorname{div}\left(2 v \mathbf{D}\left(\mathbf{v}_{\text {ref }}\right)\right)=\mathbf{0} \\
\operatorname{div} \mathbf{v}_{\text {ref }}=0,
\end{array}
$$

with the boundary conditions

$$
\begin{aligned}
\mathbf{v}_{\text {ref }} & =\mathbf{0} & & \text { on } \Gamma_{\text {wall }}, \\
\mathbf{v}_{\text {ref }} & =\mathbf{v}_{\text {in }} & & \text { on } \Gamma_{\text {in }}, \\
\mathbf{T n}-\frac{1}{2} \varrho\left(\mathbf{v}_{\text {ref }} \cdot \mathbf{n}\right)_{-} \mathbf{v}_{\text {ref }} & =-P(t) \mathbf{n} & & \text { on } \Gamma_{\text {out }},
\end{aligned}
$$

where $\mathbf{T}=-p \mathbf{I}+2 \mu \mathbf{D}\left(\mathbf{v}_{\text {ref }}\right)$. The vector $\mathbf{n}$ is the unit normal vector to the boundary $\partial \Omega$ which consists of three parts: $\Gamma_{\text {wall }}$ denotes the walls and $\Gamma_{i n}, \Gamma_{\text {out }}$ are the inlet and outlet, respectively. The dynamic viscosity is taken as a constant $\mu=3.7 \cdot 10^{-3} \frac{\mathrm{kg}}{\mathrm{ms}}$, density $\varrho=1000 \frac{\mathrm{kg}}{\mathrm{m}^{3}}$, kinematic viscosity $v=\frac{\mu}{\varrho}$. At the outlet we prescribe the pressure mean value $P(t)$ which is supposed to be based on information from measurements in the aorta as a function of time. In stenotic valves, regurgitation occurs and it can lead to instabilities. The backflow stabilization used at the outlet is studied in Braack and Mucha (2014) and Bertoglio and Caiazzo (2014). The velocity $v_{i n}$ is given as a parabolic profile with its magnitude scaled by a time dependent factor $V(t)$, i.e.

$$
v_{i n}(t, \mathbf{x})=1.5 V(t) \max \left(|\mathbf{x}-\mathcal{T}|^{2}, 0\right) / r^{2},
$$

where where $\mathcal{T}$ is the centerpoint of an input plane and $r$ is its radius for parabolic profile.

Both functions $P(t)$ and $V(t)$ depend on the stenosis severity. For the purpose of this work, we used the functions $P(t)$ and $V(t)$ with the peak velocity $v_{\max }=1.0 \mathrm{~m} / \mathrm{s}$, the peak pressure $p_{\max }=110 \mathrm{mmHg}$ and the diastolic pressure $p_{\min }=75 \mathrm{mmHg}$, see Fig. 1. For better illustration we use in the text unit millimeter of mercury while $1 \mathrm{~Pa}=$ $0,0075 \mathrm{mmHg}$. One cardiac cycle lasts $1 \mathrm{~s}$, the time when the aortic valve is considered open is $0.25 \mathrm{~s}$. The detailed description of the terms shown in Fig 1 will be provided in Part 2 of this study. 

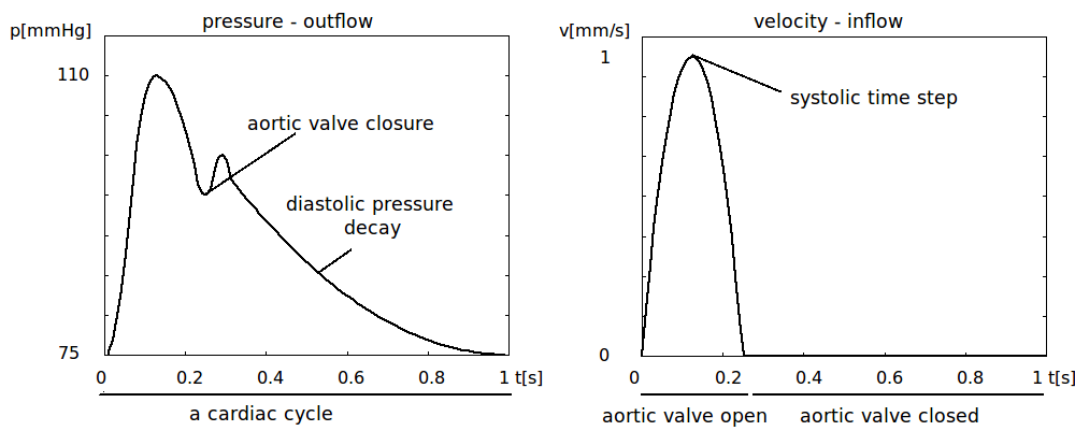

Figure 1: Dependence of the prescribed outlet pressure and inlet velocity functions $P$ and $V$ on the time.

The computational meshes for the reference flow are shown in Fig. 2 The first one represents an aortic valve with 55\% symmetric severity where the severity is given by formula (22) as a relation between the area of the healthy part, approximated here by a circle with radius $R$, and the area of the stenotic part, approximated by a circle with radius $r$, i.e.

$$
\text { severity }=\left(1-\frac{\text { area }_{r}}{\text { area }_{R}}\right) \cdot 100 \%
$$

The length of the valve $L=2.4 \mathrm{~cm}$ and the radius of the healthy part $R=1.2 \mathrm{~cm}$. For the stenotic part the length $l=1.2 \mathrm{~cm}$ and the radius $r=0.805 \mathrm{~cm}$. The second mesh with the same $L, l$ and $R$ represents an aortic valve with $50 \%$ non-symmetric severity. 

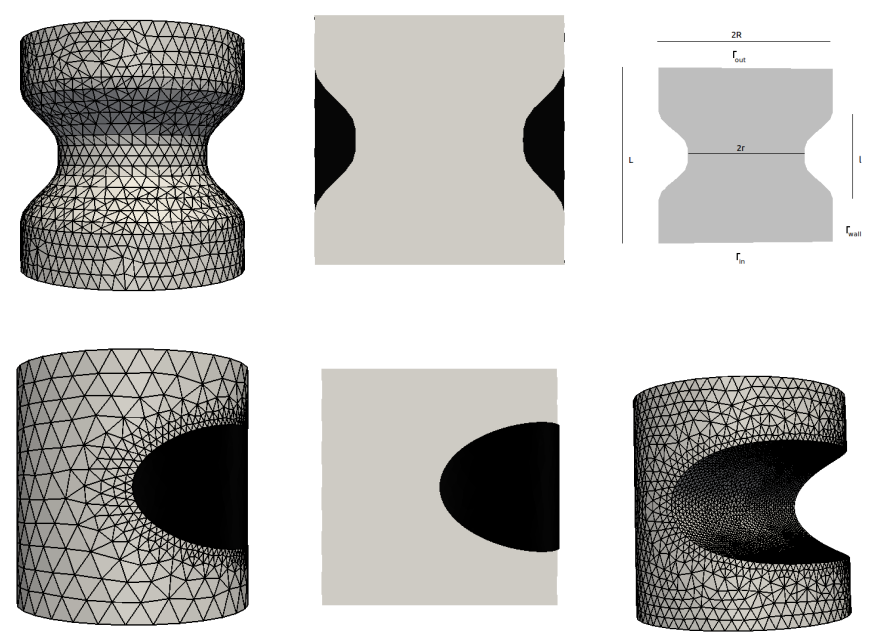

Figure 2: The first row: three-dimensional mesh for reference flow representing the valve with the symmetric stenosis with 55\% severity, for illustration also the slices of the computational mesh. Grey parts represent the domain of valve, black parts represent the tissue causing the stenosis. The second row: three-dimensional mesh for reference flow representing the valve with the $50 \%$ non-symmetric stenosis, then slice of a domain and another view to the mesh. The sizes of the meshes: $\mathrm{L}=2.4 \mathrm{~cm}, 1=1.2 \mathrm{~cm}$, $\mathrm{R}=1.2 \mathrm{~cm}, \mathrm{r}_{\mathrm{sym}}=0.805 \mathrm{~cm}$.

The resulting reference pressure $p_{\text {ref }}$ and velocity field $\mathbf{v}_{\text {ref }}$ are shown on the slice of the mesh in Fig. 3 The maximal local Reynolds numbers for these two problems, in symmetric and nonsymmetric domains, are 560 and 765 , respectively. 

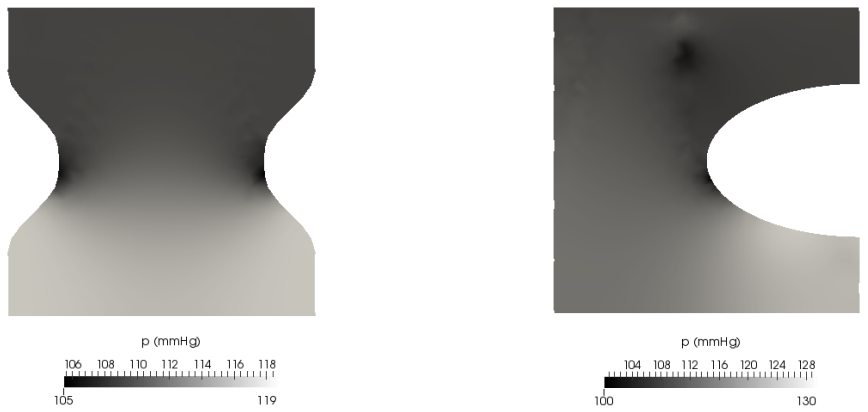

$p(\mathrm{mmHg})$
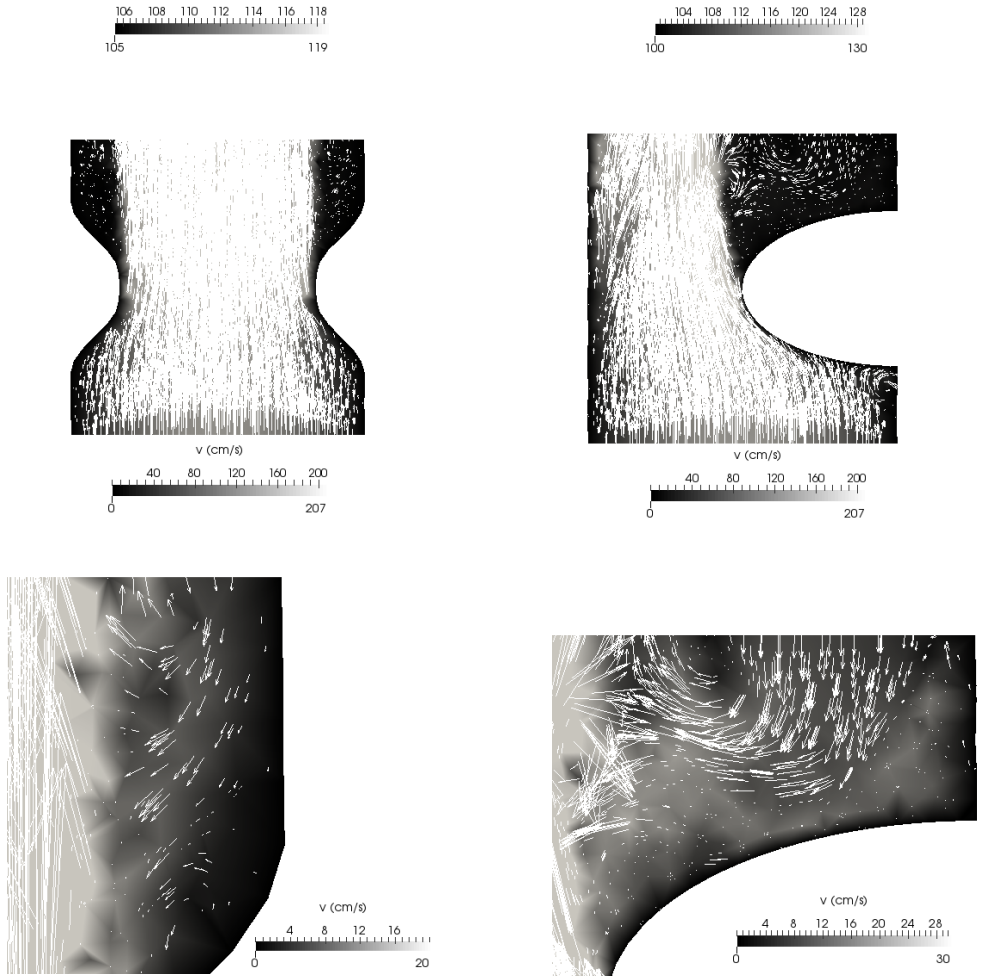

Figure 3: The first two rows depict the distribution of the reference pressure $p_{\text {ref }}$ and the reference velocity $\mathbf{v}_{\text {ref }}$ for symmetric (left column) and non-symmetric (right column) domains; the third row contains the detail of the velocity field $\mathbf{v}_{\text {ref }}$ on slice near the outlet to show the regurgitation in flow.

In the next section, we will compare the pressures $q_{\text {ppe }}$ and $q_{\text {ste }}$ obtained by the two methods proposed in Sec. 1.1.1 and 1.1.2 with this reference pressure $p_{\text {ref }}$. To do that, we will compute the pressure as an integral over the slices along the centerline, see eq. (23). The centerline is an axis passing through the vessel, $\Gamma$ denotes the cross-section area along the centerline, see Fig. 4 . The resulting curves presenting the pressure drop 
within the domain, computed through the formula (23), are shown in the Fig. 5. The centerline here passes through the $\mathrm{z}$ axis so we will use the term " $\mathrm{z}$ coordinate" for the figures, not "cross-section area along the centerline".

$$
p_{\mathrm{REF}}=\frac{\int_{\Gamma} p_{\text {ref }} d S}{\operatorname{area}(\Gamma)}
$$

We define similarly $q_{\mathrm{PPE}}$ and $q_{\mathrm{STE}}$ for $q_{\mathrm{Ppe}}$ and $q_{\mathrm{ste}}$ respectively. While we will ascertain pressure only in a systolic time step $t=0.125 \mathrm{~s}$ as a steady flow, we will consider the functions $p_{\mathrm{REF}}, q_{\mathrm{PPE}}$ and $q_{\mathrm{STE}}$ depend only on the $z$ coordinate.

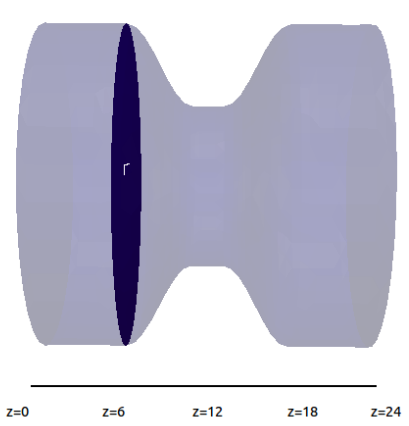

Figure 4: Cross-section areas $\Gamma$ along the centereline

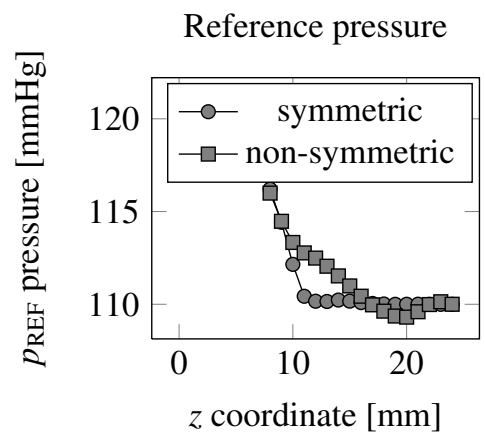

Figure 5: The reference pressure $p_{\text {REF }}$ (23) computed over cross-sections as a function of the position along the centerline for two different geometries during a systolic time step

\subsection{Comparison of the methods with regard to the ascertainment of the pressure}

In this section we will compare the results for each of the methods introduced in Sec. 1.1.1 and 1.1.2 with respect to the quality of the input velocity field. All the tests will be computed in a systolic time step $t=0.125 \mathrm{~s}$ as a steady flow, see. Fig. 6
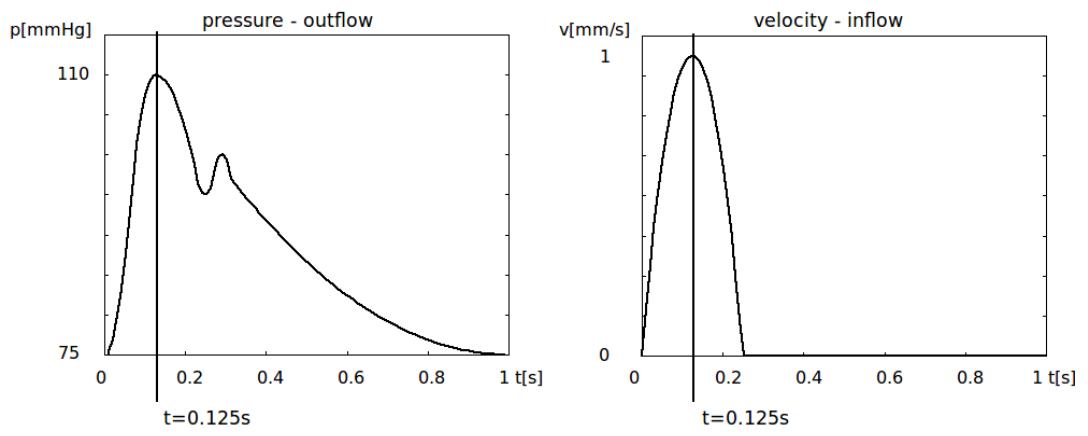

Figure 6: Systolic time step $t=0.125 \mathrm{~s}$ for computations of PPE and STE methods. 


\subsubsection{Fine data}

We will start with velocity field given on the same very fine mesh as that for which we know the velocity field exactly, i.e. velocity field known at every vertex of the mesh and with the velocity field measured exactly without noise. This case corresponds to the computation on L0 mesh, see Fig. 9 and 10.

For both cases, symmetric and non-symmetric, we compute the relative error $\frac{\left\|q_{\text {ppe }}-p_{\text {ref }}\right\|_{L^{2}}}{\left\|p_{\text {ref }}\right\|_{L^{2}}}$ and $\frac{\left\|q_{\text {ste }}-p_{\text {ref }}\right\|_{L^{2}}}{\left\|p_{\text {ref }}\right\|_{L^{2}}}$ where obtained pressures $q_{\text {ppe }}$ and $q_{\text {ste }}$ were linearly interpolated to the finer mesh where $p_{\text {ref }}$ was computed. The relative errors are shown in Tab. 1 and the point-wise errors are plotted in Fig. 2.2.1

While we are fixing on the outlet the pressures $p_{\mathrm{REF}}=q_{\mathrm{PPE}}=q_{\mathrm{STE}}$, computed through the formula (23), we are interested of comparison of the pressure drops $q_{\mathrm{PPE}}^{\text {drop }}:=$ $q_{\mathrm{PPE}}($ inlet $)-q_{\mathrm{PPE}}($ outlet $)$ and $q_{\mathrm{STE}}^{\mathrm{drop}}:=q_{\mathrm{STE}}($ inlet $)-q_{\mathrm{STE}}($ outlet $)$ compared to the reference pressure drop $p_{\mathrm{REF}}^{\text {drop }}:=p_{\mathrm{REF}}($ inlet $)-p_{\mathrm{REF}}($ outlet $)$.

The pressures $p_{\mathrm{REF}}, q_{\mathrm{PPE}}$ and $q_{\mathrm{STE}}$ are shown in the Fig. 8 and the differences between the $q_{\mathrm{PPE}}^{\text {drop }}$ and $q_{\mathrm{STE}}^{\text {drop }}$ in comparison with $p_{\mathrm{REF}}^{\text {drop }}$ are shown in Tab. 2 .
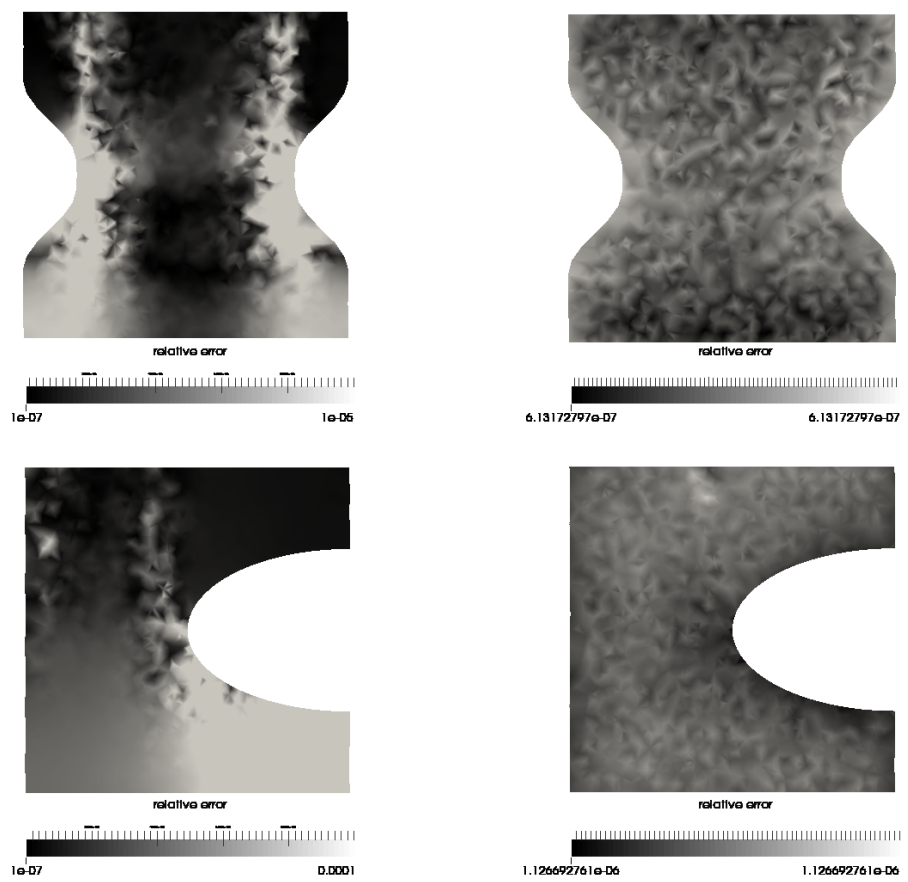

Figure 7: Errors $\left|q_{\text {ppe }}-p_{\text {ref }}\right|$ (left column) and $\left|q_{\text {ste }}-p_{\text {ref }}\right|$ (right column) in mmHg on a slice of the domain. 


\begin{tabular}{|c|c|c|}
\hline & $\frac{\| q_{\text {ppe }}-p_{\text {ref }}}{\| p_{L^{2}}} \|_{L^{2}}$ & $\frac{\| q_{\text {ste }}-p_{\text {ref }}}{\|\|_{L^{2}}}$ \\
\hline symmetric & $6.40 \mathrm{e}-04$ & $1.50 \mathrm{e}-14$ \\
\hline non-symmetric & $3.50 \mathrm{e}-03$ & $1.16 \mathrm{e}-14$ \\
\hline
\end{tabular}

Table 1: Relative errors for fine data.

L0 mesh for symmetric case without noise

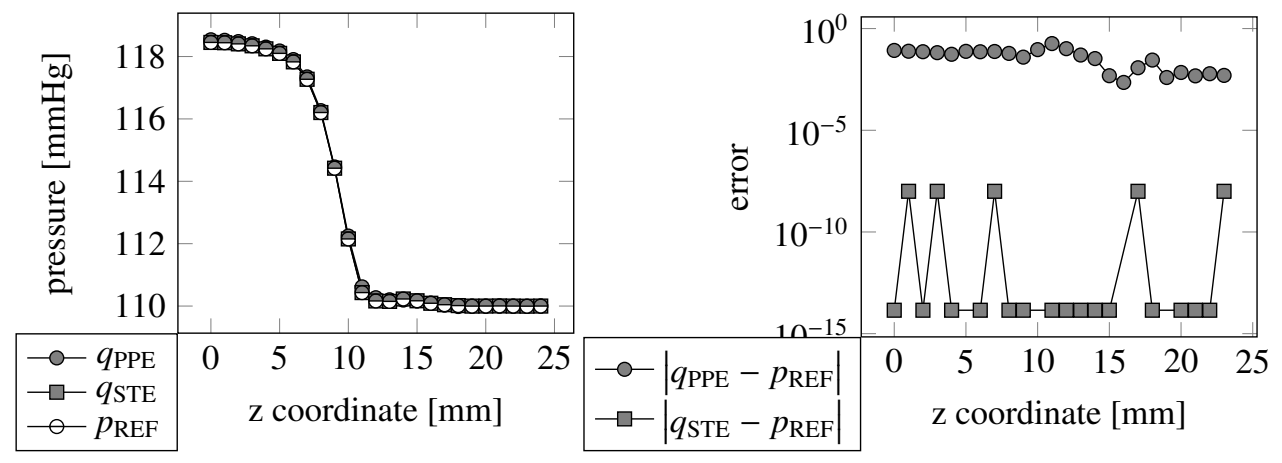

L0 mesh for non-symmetric case without noise

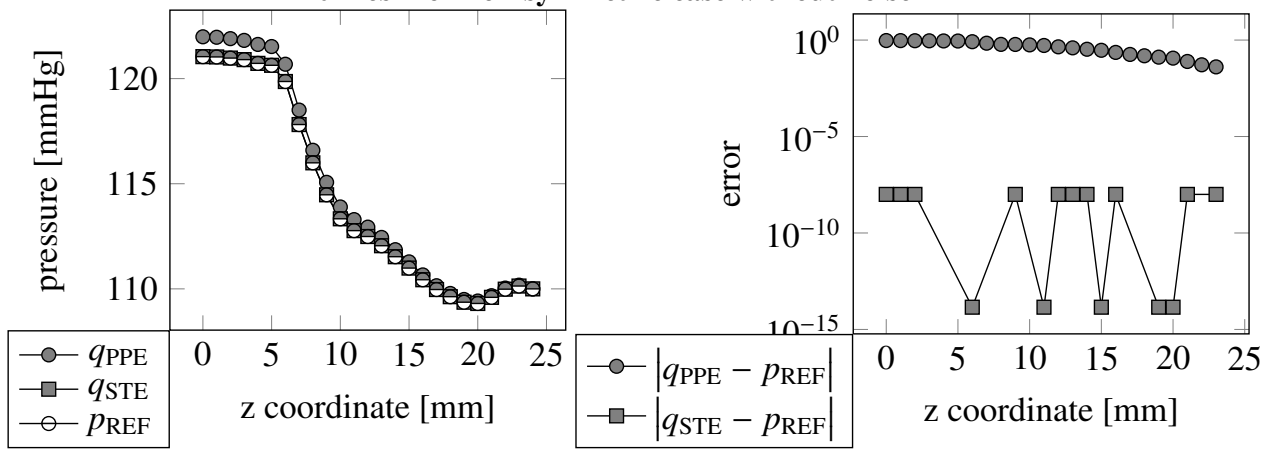

Figure 8: Results on L0 mesh without noise. First column: The reference and obtained pressure computed through the formula 23. Second column: The absolute value of difference between the reference and obtained pressure.

\begin{tabular}{|c|c|c|}
\hline & $p_{\mathrm{REF}}^{\text {drop }}-q_{\mathrm{PPE}}^{\text {drop }}$ & $p_{\mathrm{REF}}^{\text {drop }}-q_{\mathrm{STE}}^{\text {drop }}$ \\
\hline symmetric & $8.42 \mathrm{e}-02$ & $1.42 \mathrm{e}-14$ \\
\hline non-symmetric & $9.44 \mathrm{e}-01$ & $1.00 \mathrm{e}-08$ \\
\hline
\end{tabular}

Table 2: Errors in the pressure drops for fine data. 


\subsubsection{Coarse data}

In this section we will do more tests with less accurate velocity fields with fewer points where we pretend to know the velocity exactly. This means computations on the coarser meshes. All of these meshes, reffered here as a level L0 for the finest mesh and L4 for the coarsest mesh, are shown in Fig. 9 and 10 .

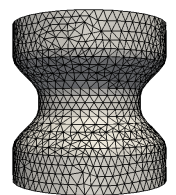

L0 mesh: 19.861 nodes
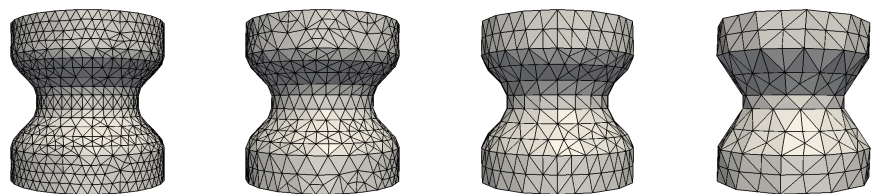

L1 mesh: 9.883 L2 mesh: 4.856 L3 mesh: 2.405 L4 mesh: 1.138 nodes nodes

Figure 9: The computational mesh L0 for the reference flow (16) and the coarser meshes for ascertaining pressure for symmetric case

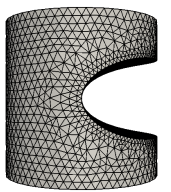

L0 mesh:

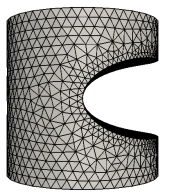

L1 mesh:
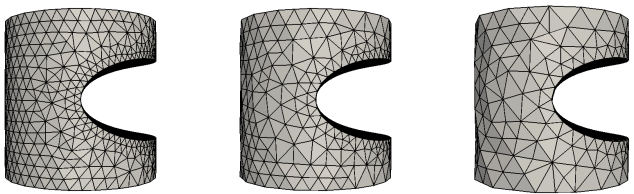

22.427 nodes

L2 mesh: 6.255 L3 mesh: 3.085 L4 mesh: 1.479

nodes

nodes

nodes

Figure 10: The computational mesh L0 for the reference flow (16) and the coarser meshes for ascertaining pressure for non-symmetric case

We also compute the relative errors $\frac{\left\|q_{\text {ppe }}-p_{\text {ref }}\right\|_{L^{2}}}{\| p_{\text {ref }} L_{L^{2}}}$ and $\frac{\left\|q_{\text {sts }}-p_{\text {ref }}\right\|_{L^{2}}}{\left\|p_{\text {ref }}\right\|_{L^{2}}}$ for these five types of meshes. The results are shown in Tab. 3. the convergence curves for symmetric and non-symmetric case are shown in Fig. 11] $1 / h$ here is taken as cube root of the number of nodes of the mesh.

Pressures $p_{\mathrm{REF}}, q_{\mathrm{PPE}}$ and $q_{\mathrm{STE}}$ and also the errors $\left|q_{\mathrm{PPE}}-p_{\mathrm{REF}}\right|$ are plotted in Fig 14 19. The errors in pressure drop estimations are shown in Tab. 4 

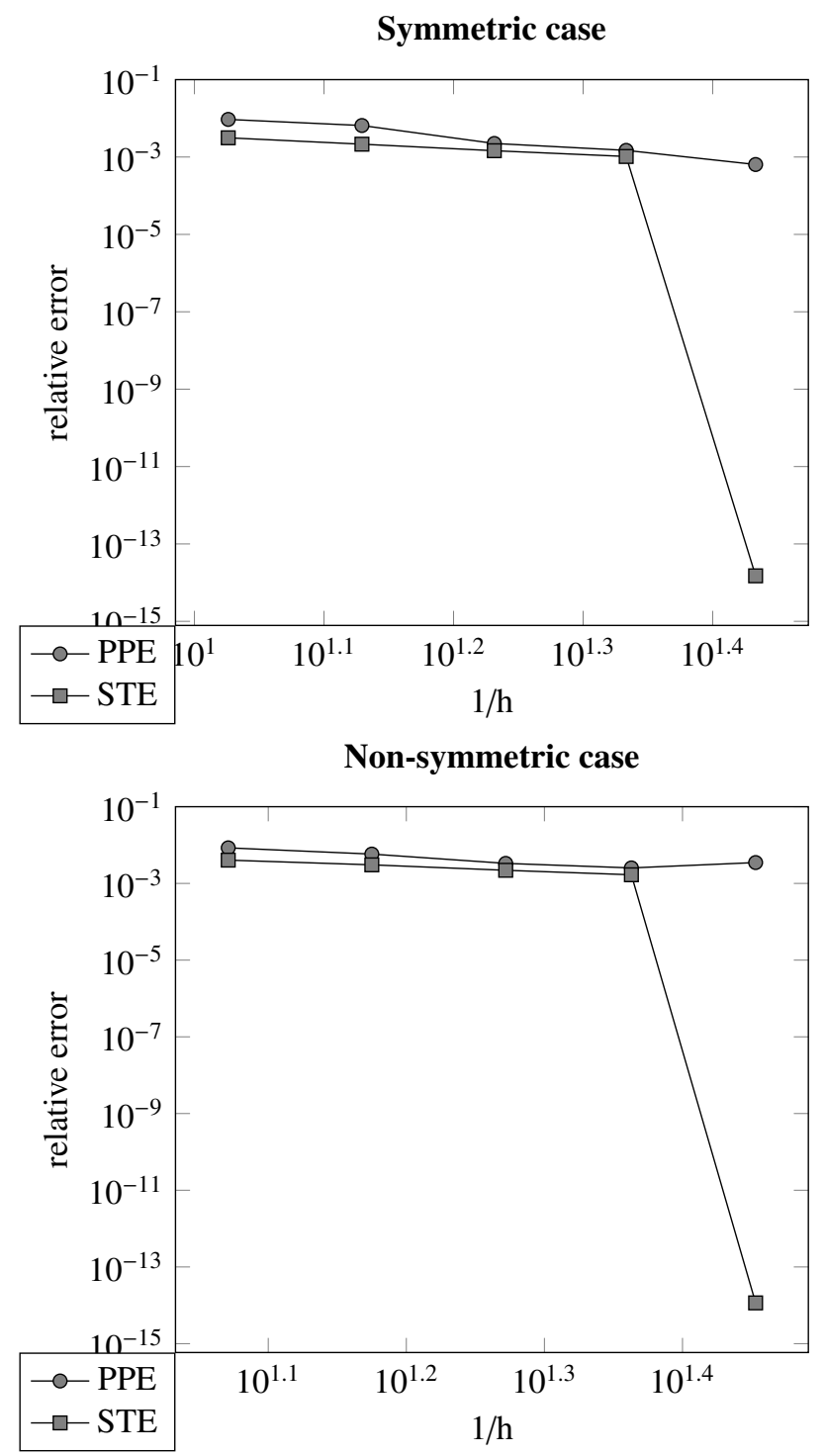

Figure 11: Convergence curves of relative errors for coarse data without noise. 


\begin{tabular}{|c|c|c|c|c|c|c|}
\hline & \multicolumn{3}{|c|}{ symmetric case } & \multicolumn{3}{c|}{ non-symmetric case } \\
\hline & $\frac{\mid q \mathrm{PPE}-p_{L^{2}}}{|p|_{L^{2}}}$ & $\frac{\left|q_{S \mathrm{ST}}-p\right|_{L^{2}}}{|p|_{L^{2}}}$ & ratio & $\frac{|q \mathrm{PPE}-p|_{L^{2}}}{|p|_{L^{2}}}$ & $\frac{\mid q_{S \mathrm{STE}}-p_{L^{2}}}{|p|_{L^{2}}}$ & ratio \\
\hline L0N0 & $6.40362 \mathrm{e}-04$ & $1.49962 \mathrm{e}-14$ & - & $3.49798 \mathrm{e}-03$ & $1.15522 \mathrm{e}-14$ & - \\
\hline L1N0 & $1.48584 \mathrm{e}-03$ & $1.03299 \mathrm{e}-03$ & 1.438 & $2.53496 \mathrm{e}-03$ & $1.67932 \mathrm{e}-03$ & 1.510 \\
\hline L2N0 & $2.24117 \mathrm{e}-03$ & $1.45550 \mathrm{e}-03$ & 1.540 & $3.32938 \mathrm{e}-03$ & $2.19963 \mathrm{e}-03$ & 1.514 \\
\hline L3N0 & $6.52214 \mathrm{e}-03$ & $2.14646 \mathrm{e}-03$ & 3.039 & $5.81674 \mathrm{e}-03$ & $3.05025 \mathrm{e}-03$ & 1.907 \\
\hline L4N0 & $9.37379 \mathrm{e}-03$ & $3.13883 \mathrm{e}-03$ & 2.986 & $8.45922 \mathrm{e}-03$ & $4.04690 \mathrm{e}-03$ & 2.090 \\
\hline \hline L0N5 & $9.03476 \mathrm{e}-04$ & $6.66165 \mathrm{e}-04$ & 1.356 & $4.91294 \mathrm{e}-03$ & $2.80946 \mathrm{e}-03$ & 1.749 \\
\hline L1N5 & $1.81613 \mathrm{e}-03$ & $1.25684 \mathrm{e}-03$ & 1.445 & $4.94317 \mathrm{e}-03$ & $3.33793 \mathrm{e}-03$ & 1.481 \\
\hline L2N5 & $2.40288 \mathrm{e}-03$ & $1.52389 \mathrm{e}-03$ & 1.577 & $4.84234 \mathrm{e}-03$ & $3.82561 \mathrm{e}-03$ & 1.266 \\
\hline L3N5 & $7.10268 \mathrm{e}-03$ & $2.49039 \mathrm{e}-03$ & 2.852 & $8.07420 \mathrm{e}-03$ & $6.46264 \mathrm{e}-03$ & 1.249 \\
\hline L4N5 & $9.14510 \mathrm{e}-03$ & $3.17592 \mathrm{e}-03$ & 2.880 & $9.87244 \mathrm{e}-03$ & $8.45561 \mathrm{e}-03$ & 1.168 \\
\hline \hline L0N10 & $1.45388 \mathrm{e}-03$ & $1.32869 \mathrm{e}-03$ & 1.094 & $6.89162 \mathrm{e}-03$ & $5.62814 \mathrm{e}-03$ & 1.224 \\
\hline L1N10 & $2.37458 \mathrm{e}-03$ & $1.74884 \mathrm{e}-03$ & 1.358 & $8.12804 \mathrm{e}-03$ & $6.08856 \mathrm{e}-03$ & 1.335 \\
\hline L2N10 & $2.86221 \mathrm{e}-03$ & $1.83955 \mathrm{e}-03$ & 1.556 & $7.75631 \mathrm{e}-03$ & $6.69936 \mathrm{e}-03$ & 1.158 \\
\hline L3N10 & $7.81501 \mathrm{e}-03$ & $3.12834 \mathrm{e}-03$ & 2.498 & $1.19152 \mathrm{e}-02$ & $1.20042 \mathrm{e}-02$ & 0.993 \\
\hline L4N10 & $9.06467 \mathrm{e}-03$ & $3.44484 \mathrm{e}-03$ & 2.631 & $1.55605 \mathrm{e}-02$ & $1.57420 \mathrm{e}-02$ & 0.988 \\
\hline
\end{tabular}

Table 3: Relative errors for PPE and STE methods.

\begin{tabular}{|c|c|c|c|c|c|c|}
\hline & \multicolumn{3}{|c|}{ symmetric case } & \multicolumn{3}{c|}{ non-symmetric case } \\
\hline & $\left|p_{\mathrm{REF}}^{\text {drop }}-q_{\mathrm{PPE}}^{\text {drop }}\right|$ & $\left|p_{\mathrm{REF}}^{\text {drop }}-q_{\mathrm{STE}}^{\text {drop }}\right|$ & ratio & $\left|p_{\mathrm{REF}}^{\text {drop }}-q_{\mathrm{PPE}}^{\text {drop }}\right|$ & $\left|p_{\mathrm{REF}}^{\text {drop }}-q_{\mathrm{STE}}^{\text {drop }}\right|$ & ratio \\
\hline L0N0 & 0.08418611 & 0.00000000 & - & 0.94382822 & 0.00000001 & - \\
\hline L1N0 & 0.23119261 & 0.04775308 & 4.841 & 0.44089585 & 0.11688687 & 3.772 \\
\hline L2N0 & 0.32041433 & 0.04229078 & 7.576 & 0.50084404 & 0.09621437 & 5.206 \\
\hline L3N0 & 1.19112938 & 0.13581371 & 8.770 & 1.00590735 & 0.03276853 & 30.697 \\
\hline L4N0 & 1.86391129 & 0.26864459 & 6.938 & 1.41908330 & 0.14511580 & 9.779 \\
\hline \hline L0N5 & 0.04192082 & 0.10039530 & 0.418 & 1.16712177 & 0.29000982 & 4.024 \\
\hline L1N5 & 0.33371535 & 0.06318706 & 5.281 & 0.99928618 & 0.17859268 & 5.595 \\
\hline L2N5 & 0.23331512 & 0.13818813 & 1.688 & 0.71064417 & 0.01646946 & 43.149 \\
\hline L3N5 & 1.35402752 & 0.27655645 & 4.896 & 1.63957690 & 1.18700155 & 1.381 \\
\hline L4N5 & 1.89644339 & 0.35499218 & 5.342 & 0.21993680 & 1.43692624 & 0.153 \\
\hline \hline L0N10 & 0.00337416 & 0.19589881 & 0.017 & 1.39336416 & 0.58381577 & 2.387 \\
\hline L1N10 & 0.43341550 & 0.16972165 & 2.554 & 1.55473599 & 0.46915924 & 3.314 \\
\hline L2N10 & 0.14353863 & 0.23876727 & 0.601 & 0.91691954 & 0.05801561 & 15.805 \\
\hline L3N10 & 1.51290953 & 0.41190733 & 3.673 & 2.27005748 & 2.40155599 & 0.945 \\
\hline L4N10 & 1.92480230 & 0.43722139 & 4.402 & 1.86438319 & 3.02559840 & 0.616 \\
\hline
\end{tabular}

Table 4: Error in the pressure drop for PPE and STE methods.

\subsubsection{Data with the noise}

In this section we want to simulate the fact that velocity is measured with the error or noise. While there is an interpolation error due to the limited amount of points where 
we know the velocity, we will also include the error of the measurement which can be as much as $5 \%$ or $10 \%$. This means that the velocity $\mathbf{v}_{\text {ref }}(\overline{\mathbf{x}})$ in the point $\overline{\mathbf{x}}$ is replaced by the $\mathbf{v}_{\text {meas }}(\overline{\mathbf{x}})=(1 \pm \varepsilon(\overline{\mathbf{x}})) \mathbf{v}_{\text {ref }}(\overline{\mathbf{x}})$ where $\varepsilon(\overline{\mathbf{x}}) \in[0,0.05]$ for maximal 5\% error, $\varepsilon(\overline{\mathbf{x}}) \in[0,0.1]$ for maximal $10 \%$ error respectively. That is the vector of the reference (exact) velocity is (due to the measurement) lessened or increased in magnitude by the error $5 \%$ or $10 \%$. This is numerically simulated by adding a random number $\varepsilon \in[-0.05,0.05]$, $\varepsilon \in[-0.1,0.1]$ respectively, to each point where we know the velocity.

We can combine these two problems (error due to information at a limited amount of points and error due to the deviations of measured and exact vectors). We will distinguish the meshes for computation, see Fig 9 and 10 , as levels L0-L4 and the error due to the deviation as N0, N5 and N10 for including noise $0 \%, 5 \%$ or $10 \%$.

The relative errors between the reference and obtained pressure $\frac{\left\|q_{\text {ppe }}-p_{\text {ref }}\right\|_{L^{2}}}{\left\|p_{\text {ref }}\right\|_{L^{2}}}$ and $\frac{\left\|q_{\text {ste }}-p_{\text {ref }}\right\|_{L^{2}}}{\left\|p_{\text {ref }}\right\|_{L^{2}}}$ are shown for both geometries(symmetric and non-symmetric) and for all computational meshes L0-L4 in Tab. 3 . They are also shown in Fig. 12 and 13 , 1/h here is taken as cube root of the number of nodes of the mesh.

Pressures $p_{\mathrm{REF}}, q_{\mathrm{PPE}}$ and $q_{\mathrm{STE}}$ and also the errors $\left|q_{\mathrm{PPE}}-p_{\mathrm{REF}}\right|$ are plotted in Fig 14 19. The errors in pressure drop estimations are shown in Tab.4 

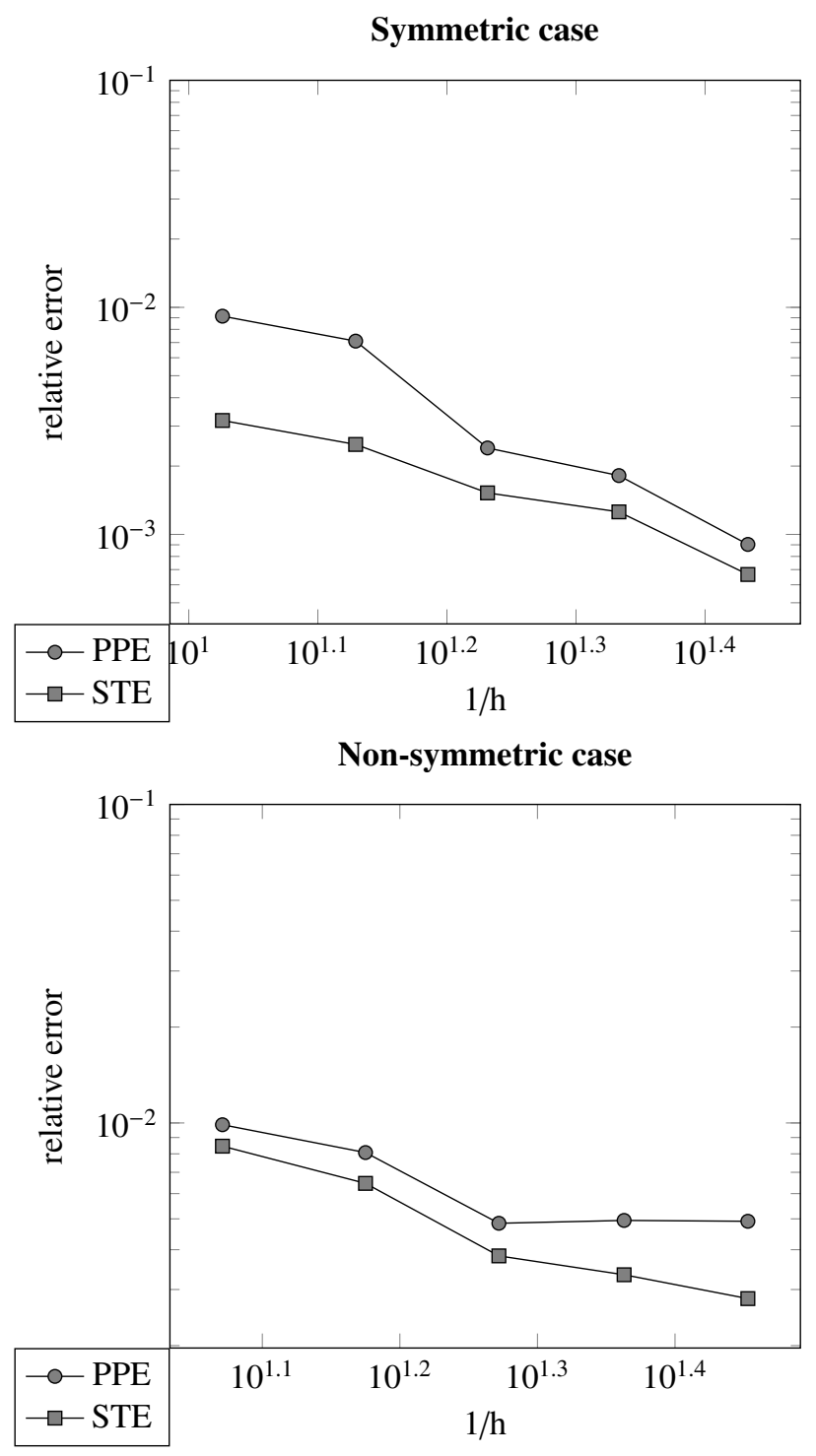

Figure 12: Convergence curves of relative errors for coarse data with 5\% noise. 

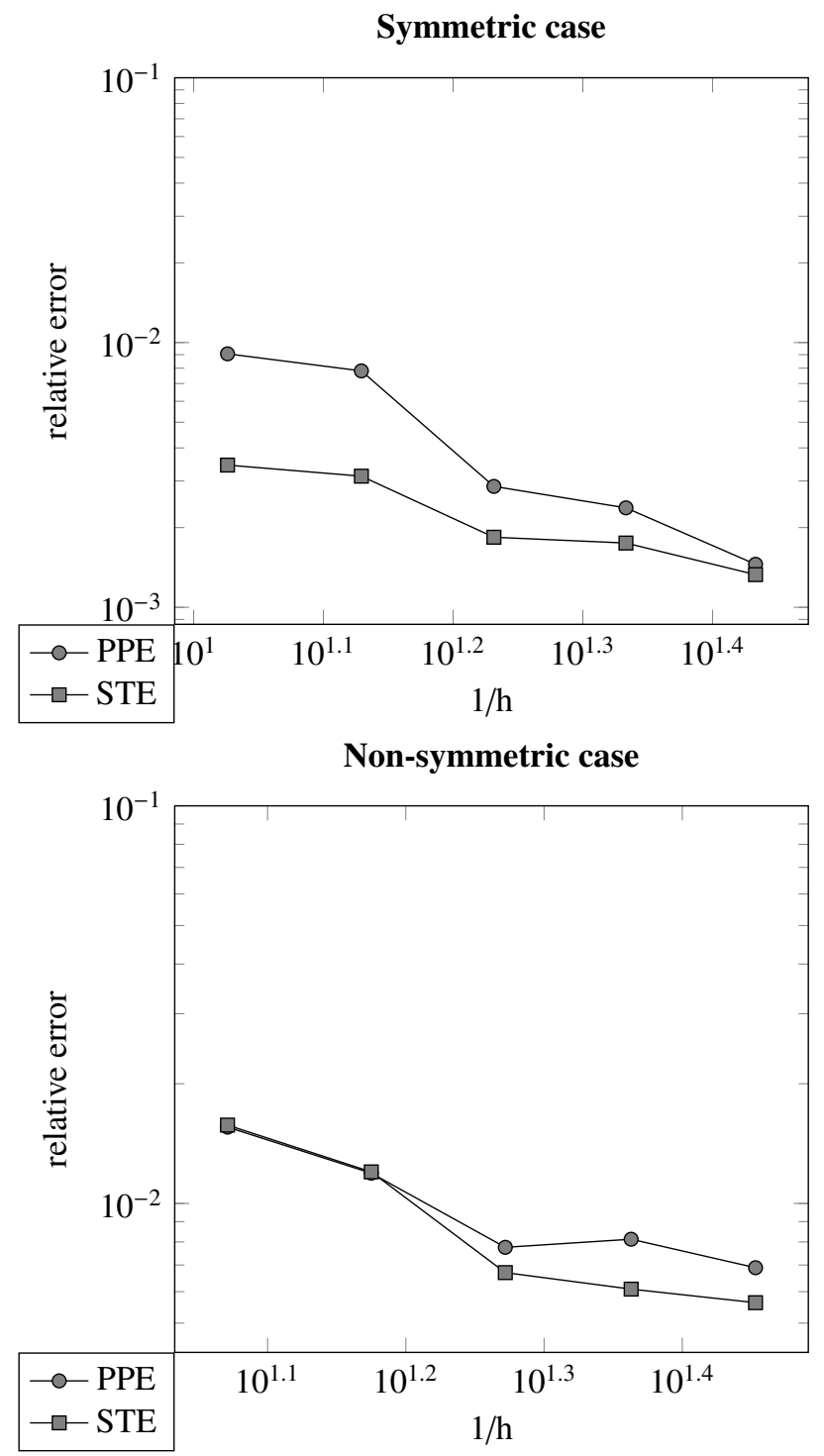

Figure 13: Convergence curves of relative errors for coarse data with $10 \%$ noise.

The reference pressure for the symmetric and non-symmetric problems is shown in Fig. 5, the obtained pressure for different problems are shown in Fig $14 \sqrt{19}$. 
L0 mesh for symmetric case without noise
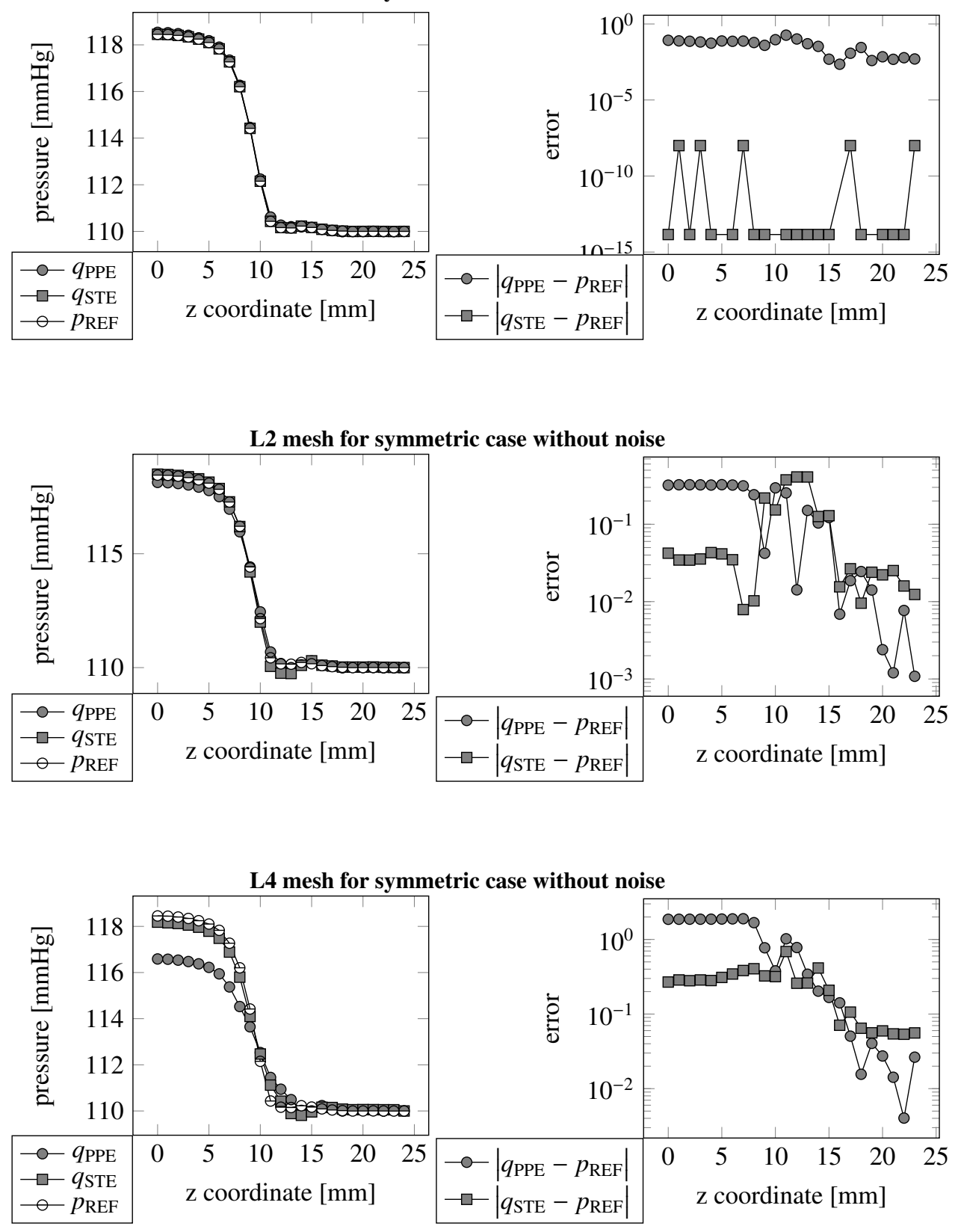

Figure 14: Reference and obtained pressure computed through the formula $(23)$ in the first column and the absolute value of difference between the reference and obtained pressure in a second column for both methods. 

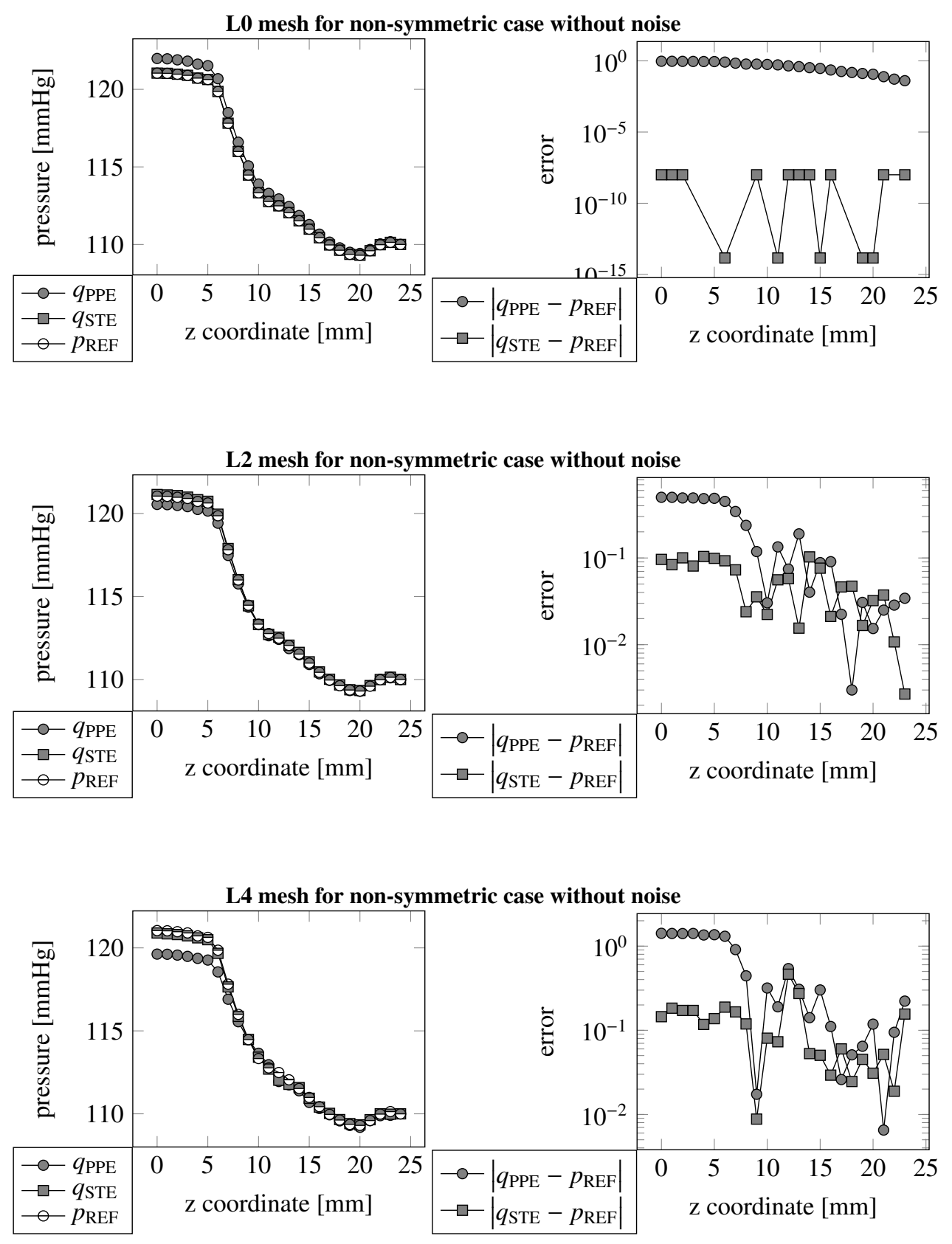

Figure 15: Reference and obtained pressure computed through the formula (23) in the first column and the absolute value of difference between the reference and obtained pressure in a second column for both methods. 

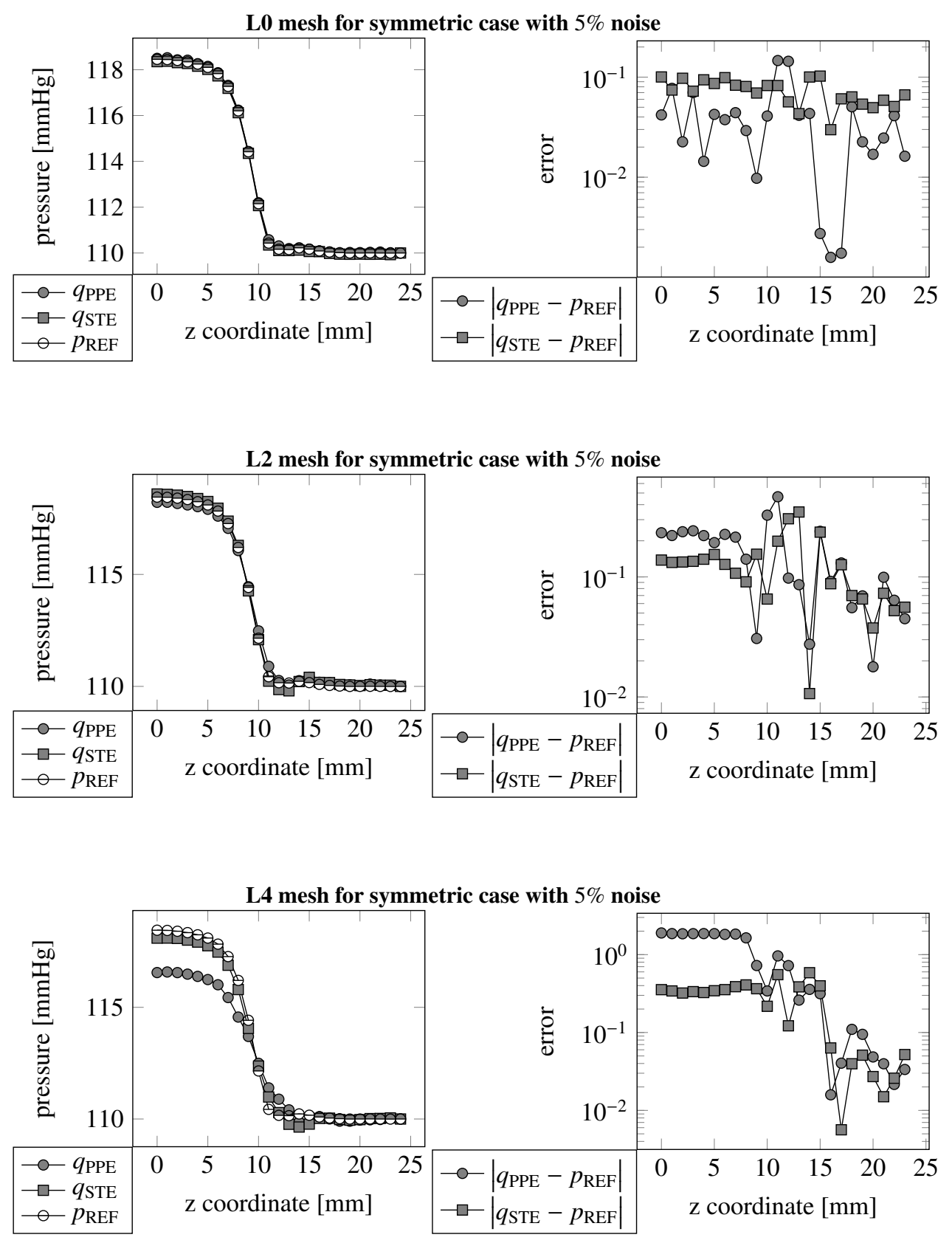

Figure 16: Reference and obtained pressure computed through the formula (23) in the first column and the absolute value of difference between the reference and obtained pressure in a second column for both methods. 

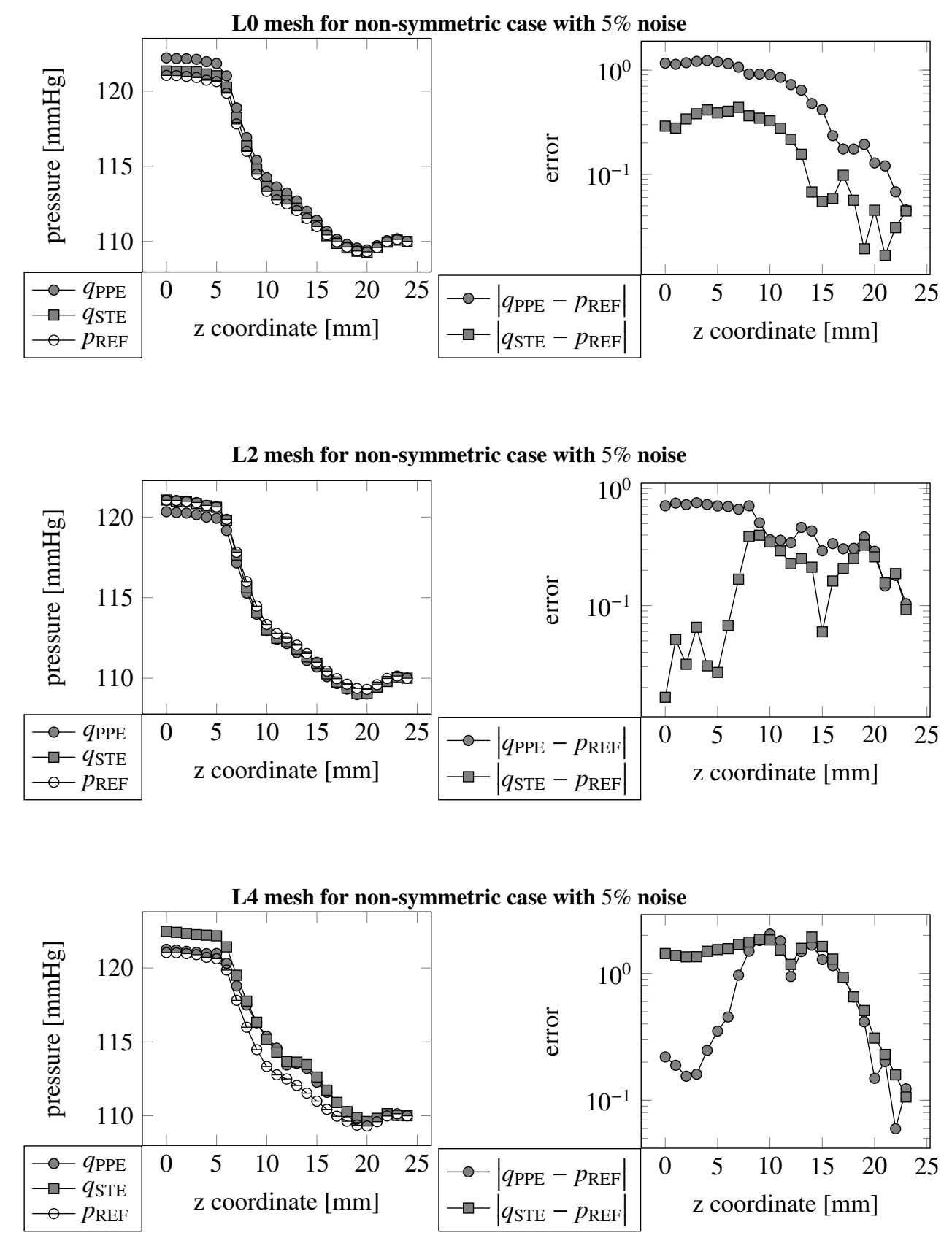

Figure 17: Reference and obtained pressure computed through the formula 23 in the first column and the absolute value of difference between the reference and obtained pressure in a second column for both methods. 
L0 mesh for symmetric case with $10 \%$ noise
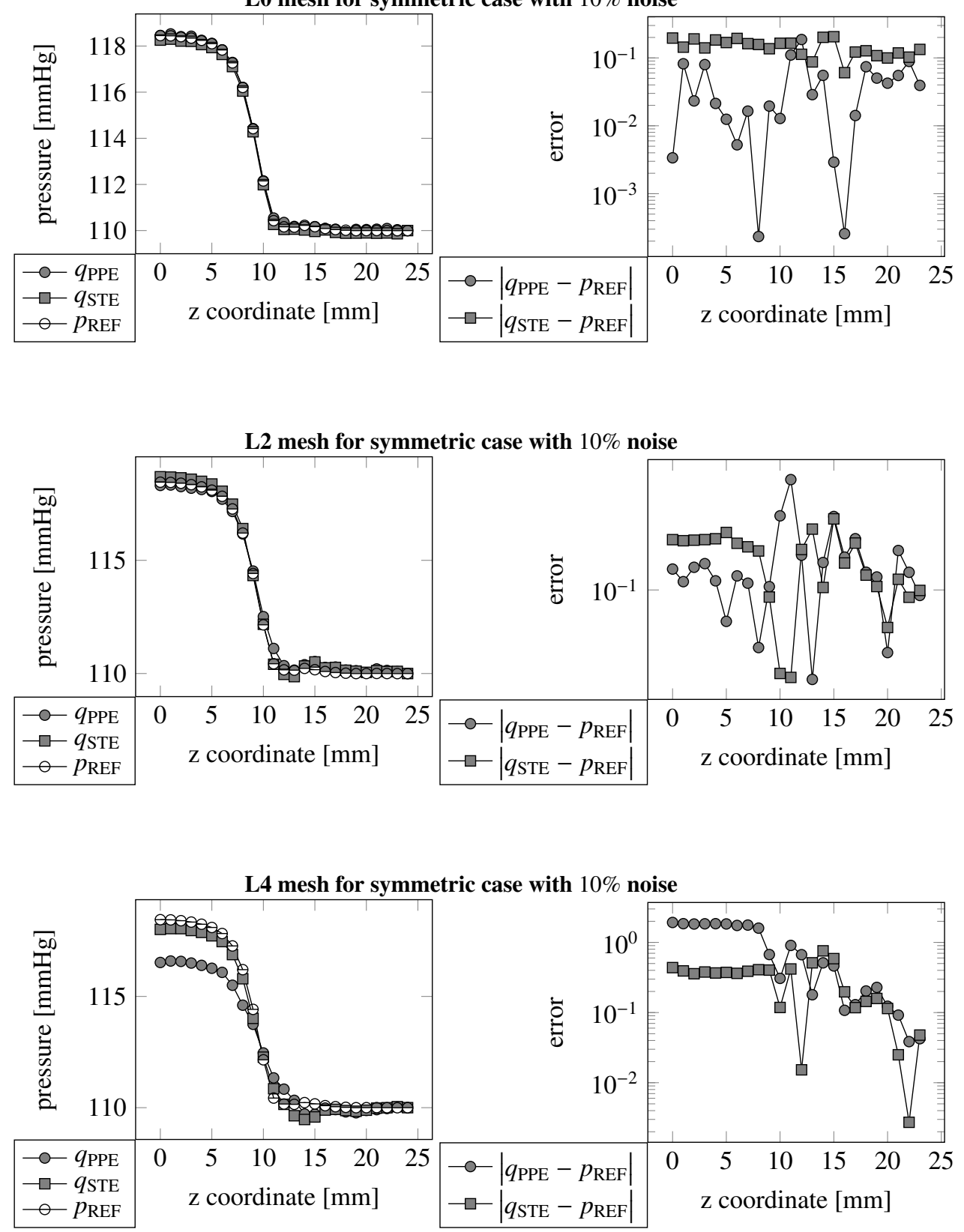

Figure 18: Reference and obtained pressure computed through the formula (23) in the first column and the absolute value of difference between the reference and obtained pressure in a second column for both methods. 
L0 mesh for non-symmetric case with $10 \%$ noise
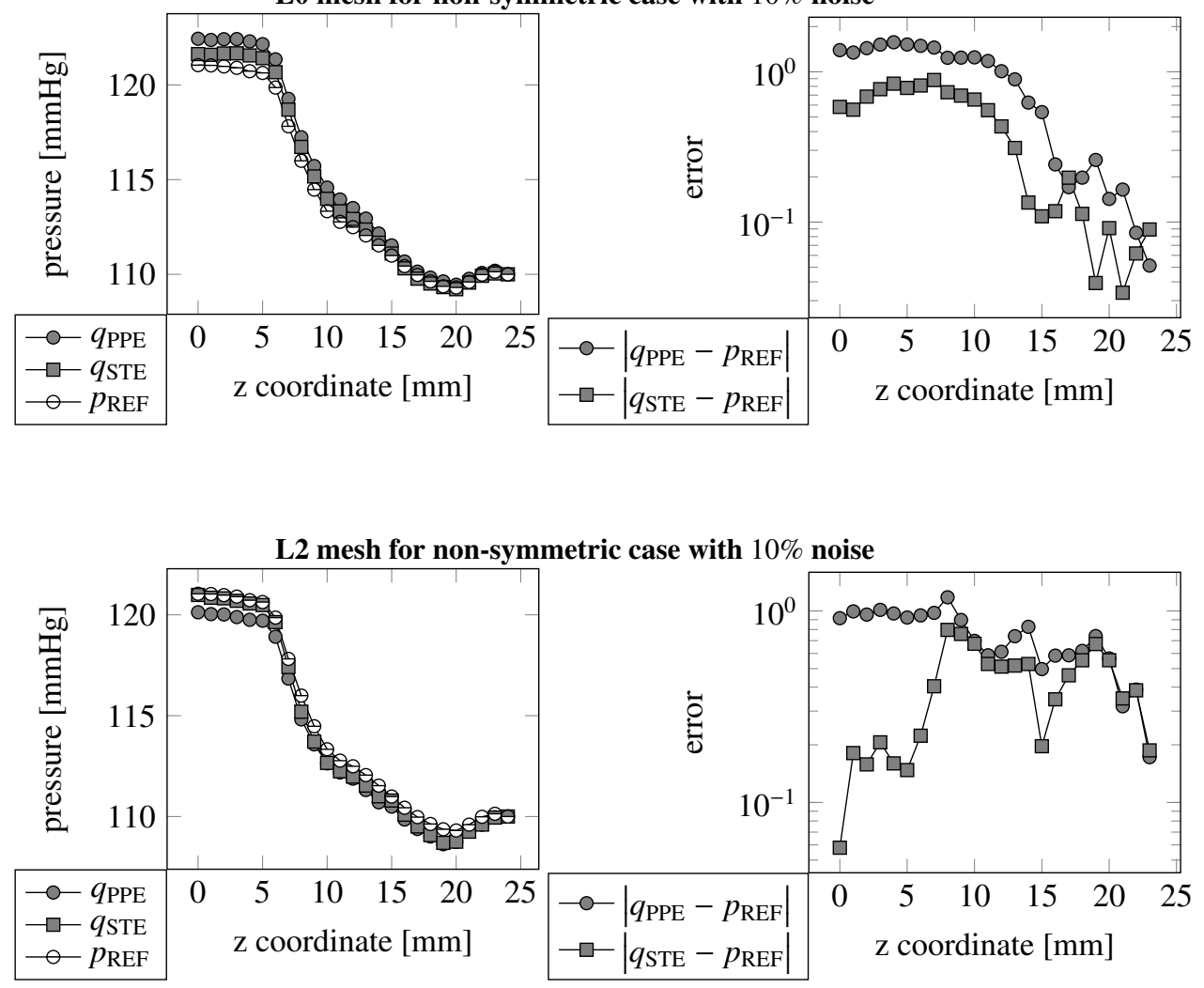

L4 mesh for non-symmetric case with $10 \%$ noise

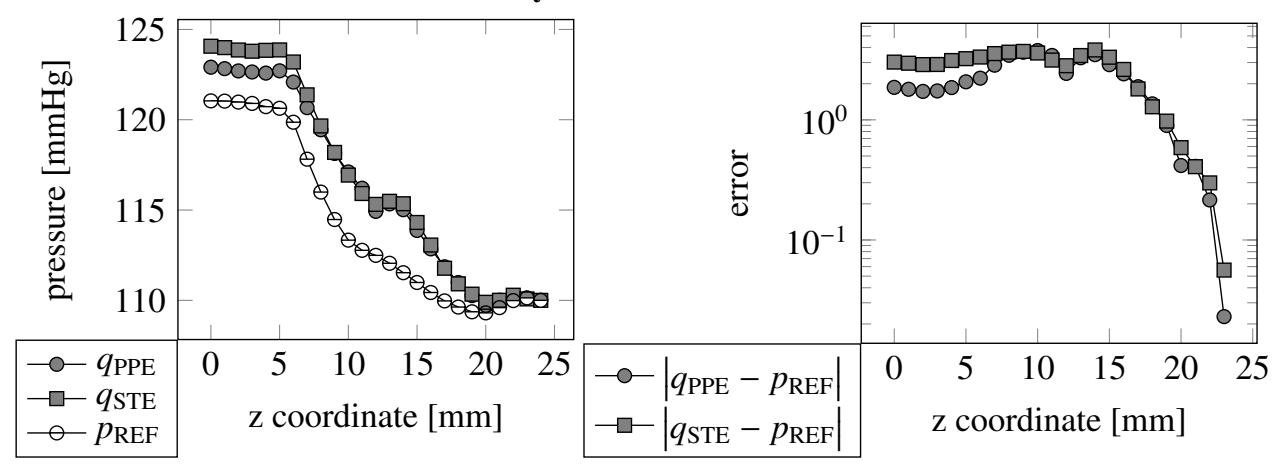

Figure 19: Reference and obtained pressure computed through the formula 23 in the first column and the absolute value of difference between the reference and obtained pressure in a second column for both methods. 


\section{Discussion}

In this study, we have developed a mathematically rigorous methodology for the determination of the pressure field from a knowledge of the velocity field in the case of the Navier-Stokes fluid, with a view towards the determination of the pressure loss across a diseased valve. In part 1 we tested the problem for idealized geometries. The study captures some of the salient features concerning the flow of blood in cardiovascular systems, such as the mechanical properties of blood and the spatiotemporal characteristics of ventricular, trans-valvular, and arterial blood flow.

Also, we incorporated the normal physiological mechanical properties of the conduit. However, fully integrating the deformable conduit with moving fluid/solid boundary into the existing model, in order to develop a comprehensive mathematical model, is considerably more difficult and will be the focus of future endeavors. Testing a more refined model against clinically relevant datasets then may be undertaken.

\section{References}

Akins, C. W., Travis, B., and Yoganathan, A. P. (2008). Energy loss for evaluating heart valve performance. The Journal of Thoracic and Cardiovascular Surgery, 136(4):820-833.

Bertoglio, C. and Caiazzo, A. (2014). A tangential regularization method for backflow stabilization in hemodynamics. Journal of Computational Physics, 261:162-171.

Bolin, C. D. and Raguin, L. G. (2008). Methodology to estimate the relative pressure field from noisy experimental velocity data. Journal of Physics: Conference Series.

Braack, M. and Mucha, P. B. (2014). Directional Do-Nothing Condition for the NavierStokes Equations. Journal of Computational Mathematics, 32(5):507-521.

Cayco, M. E. and Nicolaides, R. A. (1986). Finite Element Technique for Optimal Pressure Recovery from Stream Function Formulation of Viscous Flows. Mathematics of Computation, 46(174):371.

Charonko, J. J., King, C. V., Smith, B. L., and Vlachos, P. P. (2010). Assessment of pressure field calculations from particle image velocimetry measurements. Measurement Science and Technology.

Dabiri, J. O., Bose, S., Gemmell, B. J., Colin, S. P., and Costello, J. H. (2014). An algorithm to estimate unsteady and quasi-steady pressure fields from velocity field measurements. The Journal of Experimental Biology, 217(3):331-336.

Dasi, L. P., Simon, H. A., Sucosky, P., and Yoganathan, A. P. (2009). FLUID MECHANICS OF ARTIFICIAL HEART VALVES. Clinical and Experimental Pharmacology and Physiology, 36(2):225-237. 
Guermond, J. L., Minev, P., and Shen, J. (2006). An overview of projection methods for incompressible flows. Computer Methods in Applied Mechanics and Engineering, 195(44-47):6011-6045.

Gurka, R., Liberzon, A., Hefetz, D., and Rubinstein, D. (1999). Computation of pressure distribution using PIV velocity data. In Proceedings of the 3rd international workshop on particle image velocimetry-PIV'99.

Heys, J. J., Manteuffel, T. A., McCormick, S. F., Milano, M., Westerdale, J., and Belohlavek, M. (2010). Weighted least-squares finite elements based on particle imaging velocimetry data. Journal of Computational Physics, 229(1):107-118.

Hron, J. and Madlik, M. (2007). Fluid-structure interaction with applications in biomechanics. Nonlinear Analysis: Real World Applications, 8(5):1431-1458.

Krittian, S. B. S., Lamata, P., Michler, C., Nordsletten, D. A., Bock, J., Bradley, C. P., Pitcher, A., Kilner, P. J., Markl, M., and Smith, N. P. (2012). A finite-element approach to the direct computation of relative cardiovascular pressure from timeresolved MR velocity data. Medical Image Analysis.

Liu, J.-G., Liu, J., and Pego, R. L. (2010). Stable and accurate pressure approximation for unsteady incompressible viscous flow. Journal of Computational Physics, 229(9):3428-3453.

Logg, A., Mardal, K. A., and Wells, G. N., editors (2012). Automated Solution of Differential Equations by the Finite Element Method, volume 84 of Lecture Notes in Computational Science and Engineering. Springer Berlin Heidelberg, Berlin, Heidelberg.

Maria Denaro, F. (2003). On the application of the Helmholtz-Hodge decomposition in projection methods for incompressible flows with general boundary conditions. International Journal for Numerical Methods in Fluids, 43(1):43-69.

Prohl, A. (2008). On pressure approximation via projection methods for nonstationary incompressible Navier-Stokes equations. SIAM Journal of Numerical Analysis, 47(1):158-180.

Rajagopal, K. R. (2015). Remarks on the notion of "pressure". International journal of non-linear mechanics, 71:165-172.

Razzaq, M., Damanik, H., Hron, J., Ouazzi, A., and Turek, S. (2012). FEM multigrid techniques for fluid-structure interaction with application to hemodynamics. Applied Numerical Mathematics, 62(9):1156-1170.

Shirokoff, D. and Rosales, R. R. (2011). An efficient method for the incompressible Navier-Stokes equations on irregular domains with no-slip boundary conditions, high order up to the boundary. Journal of Computational Physics, 230(23):86198646. 
Song, S. M., Leahy, R. M., Boyd, D. P., Brundage, B. H., and Napel, S. (1994). Determining cardiac velocity fields and intraventricular pressure distribution from a sequence of ultrafast CT cardiac images. IEEE Transactions on Medical Imaging, 13(2):386-397.

Van Oudheusden, B. W. (2013). PIV-based pressure measurement. Measurement Science and Technology, 24(3):032001.

Yang, G.-Z., Kilner, P. J., Wood, N. B., Underwood, S. R., and Firmin, D. N. (1996). Computation of flow pressure fields from magnetic resonance velocity mapping. Magnetic Resonance in Medicine, 36(4):520-526. 